\title{
Understating the factors influencing yield strength on Mg alloys
}

\author{
Isaac Toda-Caraballo, Enrique I. Galindo-Nava \\ and Pedro E. J. Rivera-Díaz-del-Castillo \\ Department of Materials Science and Metallurgy, University of Cambridge \\ 27 Charles Babbage Rd, Cambridge, CB3 0FS, UK
}

\begin{abstract}
Taking the Hall-Petch relationship as a starting point, the factors contributing towards magnesium alloys strengthening are analysed, and their relative importance quantified. Solid solution strengthening is modelled employing a power-law approach. The effects of various processing schedules are reviewed, showing that these play a relatively minor role. Grain refinement effects are described employing thermodynamics and kinetic formulation via the Interdependence Theory approach. The effects of the rare earths are examined, and it is shown that their major contribution is towards grain size control, an effect often in conflict with solid solution strengthening. A computational approach is proposed, successfully modelling 104 grades reported in the literature. The approach may aid in the tailoring and design of magnesium alloys for yield strength.
\end{abstract}

Keywords: Magnesium alloys; Yield strength; Grain refinement; Computational thermodynamics; Alloy design 


\section{Introduction}

There is recent increased effort in the discovery of new alloys. A major related research programme in Europe is Accelerated Metallurgy, which is motivated by the fact that only $10 \%$ of the possible metallic ternary systems is known [1]. An important family of engineering alloys is that based on Magnesium. Magnesium is one of the most common elements on Earth, and its potential industrial applications have increased in the last fifty years due to a better understanding of its alloying behaviour with other elements. $\mathrm{Mg}$ is the third most commonly used element in structural applications, after aluminium and iron, due to its excellent specific mechanical properties [2]. Even so, its mechanical properties are far from those displayed by other alloys, and improvements are always required. $\mathrm{Mg}$ alloys display low ductility and poor cold workability; thus, the material is frequently produced by casting without further mechanical processing. To optimise Mg alloys, extensive work has been performed in understanding the role of its elemental additions. Only to mention some of them, additions of $\mathrm{Zr}$ or $\mathrm{Al}$ can dramatically reduce the as-cast grain size [3, 4], increasing of the yield strength, $\sigma_{Y}$. Zn improves castability [2], and additions of rare earth elements improve $\sigma_{Y}$ and optimise creep resistance via solid solution precipitation hardening [5]. Nevertheless, results in the literature display scattered information and are frequently confined to a single effect. Theories on grain size refinement, solid solution and grain boundary strengthening, and stored deformation in the material have been developed to explain each corresponding effect. However, these effects have never been explored in a combined manner. In addition to that, different compositions, processing and mechanical testing conditions need to be quantitatively examined. The objective of this work is to combine a number of theoretical studies to describe the mechanical response of Mg-alloys. Several strengthening mechanisms are considered simultaneously. A unified approach is produced aimed at understanding how each factor influences $\sigma_{Y}$, leading to alloy design with improved strength. The general model is based on a factor decomposition of the Hall-Petch relationship [6, 7], where each contribution is analysed and validated with experimental datasets available from the literature. Chemical composition and thermo-mechanical processing are employed as input to predict $\sigma_{Y}$, validating the model 
with 104 alloys.

\section{Modelling the contributions to yield strength}

The Hall-Petch theory [6, 7] predicts an inverse relationship between the yield strength, $\sigma_{y}$, and the mean grain size, $D_{g b}$ :

$$
\sigma_{y}=\sigma_{0}+\frac{k_{y}}{\sqrt{D_{g b}}}
$$

where $\sigma_{0}$ is the friction stress and $k_{Y}$ is the strengthening coefficient. The description of these parameters is usually by fitting to experimental data by the linear square method. Although there are a number of studies focusing on characterizing $\sigma_{0}$ and $k_{Y}$, experimental results display very scattered data. This is related to various processing techniques, chemical composition and testing methods. The aim of this study is to combine several theoretical studies to produce a unified approach with the intention to understand how each factor influences $\sigma_{Y}$, leading to alloy design with improved properties. Each parameter of Equation (1) is now examined separately.

\subsection{Friction Stress}

The friction stress $\sigma_{0}$ term is grain size independent and can be decomposed into three different contributions:

$$
\sigma_{0}=\sigma_{\text {fric }}+\sigma_{p}+\Delta \sigma_{s}
$$

$\sigma_{\text {fric }}$ is directly related to the critical resolved shear stress $\tau_{C R S S}$ of the predominant slip system. It has been found that different slip modes are activated in compression and tension. For instance, deformation in HCP under compression perpendicular to the basal poles displays a dominant basal slip activity [8]. The estimated value for basal slip in pure $\mathrm{Mg}$ of $\tau_{C R R S}$ lies around $1.5 \mathrm{MPa}[9]$, and in consequence $\sigma_{\text {fric }}$ is $\approx 5 \mathrm{MPa}$, by using the Taylor orientation factor. On the other hand, tensile deformation shows a strong pyramidal slip activity, where $\tau_{C R R S}$ is in the range of $5 \mathrm{MPa}$ [9], thus $\sigma_{\text {fric }} \approx 15 \mathrm{MPa}$. This effect can be observed as a function of grain size in Figure 1, where two sets of experiments of extruded 
pure Mg tested in tension [10] and compression [11] are depicted, showing a difference of 15 MPa between both conditions without change in $k_{y}$. In addition to this, two other conditions for as-cast Mg-0.8Zn (at\%) tensile [12] and compressive tests [13] show a difference of $10 \mathrm{MPa}$, without effect on $k_{y}$. The difference between both results is small and fits the predicted value from Bhattacharya [9].

A residual stress $\sigma_{p}$ related to the applied thermo-mechanical processing method is present. This term can be considered negligible for the as-cast material as no mechanical work is applied. A comparison between sets of experiments on pure $\mathrm{Mg}$ in as-cast [12], rolling [14] and extrusion [10] shows a constant hardening effect of rolling and extrusion of 18-22 MPa. This is depicted in Figure 2, where three sets of experiments are plotted. Such increase can be related to additional dislocation storage during processing. This term can differ under different processing techniques, such as severe plastic deformation, where additional deformation mechanisms are present.

A solid solution strengthening contribution $\Delta \sigma_{s}$ is also present. This effect has been the subject of several studies $[15,16,17,18]$. A power law relationship is assumed $\Delta \sigma_{s}=B_{i} X_{i}^{n}$, where $X_{i}$ is the solute content of element $i$ and $B_{i}$ is a constant for element $i$. Two exponents have been considered $n=1 / 2$ for Fleischer approach [16] and $n=2 / 3$ for Labusch approach [18]. Both exponents show similar agreement with experimental data [19]. It is assumed that:

$$
B_{i}=3 \mu \epsilon^{m} Z
$$

where $m=3 / 2$ for Fleischer's and $m=4 / 3$ for Labusch's, $\mu$ is the shear modulus of the alloy, $Z$ is a fitting constant and $\epsilon$ is a function of the atomic size misfit $\delta$ due to the strain field of the solute in the solvent, and the modulus misfit $\eta$ accounts for the the relative change in shear modulus. These two parameters, $\eta$ and $\delta$, are respectively dependant upon the compositional depdendence of $\mu$, the shear modulus, and $a$, the lattice parameter [5]:

$$
\eta=\frac{d \mu}{d X} \frac{1}{\mu_{M g}}=\frac{\mu_{i}-\mu_{M g}}{\mu_{M g}} \quad ; \quad \delta=\frac{d a}{d X} \frac{1}{a_{M g}}=\frac{a_{i}-a_{M g}}{a_{M g}}
$$


where $\mu_{M g}$ and $\mu_{i}$ are the shear modulus of Mg and element $i$ respectively, and $a_{M g}$ and $a_{i}$ are the atomic size of $\mathrm{Mg}$ and element $i$. The final expression for $\epsilon$ is $[16,20]$ :

$$
\epsilon=\left(\eta^{\prime 2}+\alpha^{2} \delta^{2}\right)^{1 / 2} \approx\left|\eta^{\prime}\right|+\alpha|\delta|
$$

where $\eta^{\prime}=\eta /(1+0.5|\eta|)$ and $\alpha$ accounts for the difference in the interaction forces between the screw and edge dislocations, respectively, and the solute atom [21]. It is generally accepted that $3<\alpha<16$ is for screw dislocations and $\alpha>16$ is for edge dislocations [22].

Irrespective of the model, it is generally assumed that the influence of solute content on yield strength scales with $\delta$ and $\eta^{\prime}$. Both parameters are shown in Figure 3 for several elements. A systematic study of the effect of the critical resolved shear stress in Mg alloys with additions of $\mathrm{Al}, \mathrm{Cd}, \mathrm{In}, \mathrm{Li}, \mathrm{Pb}, \mathrm{Tl}$ and $\mathrm{Zn}$ was carried out by Akhtar [22]. The largest hardening effect was for $\mathrm{Al}$ and $\mathrm{Zn}$, followed by $\mathrm{Pb}$, where the elements $\mathrm{Li}, \mathrm{Cd}, \mathrm{Tl}$ and In showed little contribution. A similar conclusion was reached by Lukáč [20], although the hardening effect of $\mathrm{Cd}$ was larger than $\mathrm{In}$, and close to $\mathrm{Al}$ and $\mathrm{Zn}$. Such behaviour is highlighted in Figure 3, where the value of $\delta_{M g}$ and $\eta_{M g}^{\prime}$ of each element is depicted; here $\delta_{M g}$ (bottom axis) and $\eta_{M g}^{\prime}$ (left axis) correspond to $\delta$ and $\eta^{\prime}$ of Equation (5) with respect to $\mathrm{Mg}$. In this figure, the grey squares correspond to atoms with negligible effect and grey circles to atoms with larger effect. In both works, $\alpha=16$ was taken for Labusch approximation, which considers screw dislocations as dominant, as expected for low temperature dislocations behaviour in HCP [23]. In addition to this, it has been extensively reported a large solid solution strengthening effect from rare earth (RE) elements, such as $\mathrm{Y}, \mathrm{Gd}, \mathrm{Ce}, \mathrm{Nd}$ and $\mathrm{Yb}$ $[24,5,25,26,27,28]$, which are displayed with grey rhombi in Figure 3. This extraodinary effect is not explained by the solid solution hardening models. We reached the same conclusion by studying experimental observations in $\mathrm{Mg}-\mathrm{Zn}$ [29, 12, 13, 30], Mg-Al [29, 19], Mg-Sn [31] and systems with RE elements, such as $\mathrm{Mg}-\mathrm{Y}[5,32,11]$ and $\mathrm{Mg}$-Gd [33]. The increase in yield strength produced by the additions of RE elements is substantially higher than the effect of $\mathrm{Zn}, \mathrm{Al}$ and $\mathrm{Sn}$, in spite of $\left|\delta_{M g}\right|$ being similar.

A similar effect can be observed for $\mathrm{Cu}$ alloys [21] by studying the additions of $\mathrm{Al}, \mathrm{Au}, \mathrm{Cd}$, 
Ga, Ge, In, Mg, Ni, Si and Zn, where a remarkable increase in solid solution strengthening is achieved with $\mathrm{Cd}$, In and $\mathrm{Mg}$. Figure 3 shows the corresponding values of $\delta_{C u}$ (top axis) and $\eta_{C u}^{\prime}$ (right axis) for different elements. As shown in Figure 3, the exceptions lie on the elements with a positive value of $\delta_{i}$. For Mg alloys, $\delta_{M g}>0.1$ this corresponds to Y, Gd, Ce, $\mathrm{Nd}$ and $\mathrm{Yb}$, and for $\mathrm{Cu}$ alloys $\delta_{C u}>0.15$ corresponds to $\mathrm{Cd}$, In and $\mathrm{Mg}$. This suggests that the sign of atomic size misfit induces a different behaviour on yield strength. A positive value of $\delta$ is related with compressive strains around the solute atom, which act as strong barriers for moving dislocations. A negative $\delta$ value would produce tensile strains whose effect seems to be more moderate than their compressive counterparts, according to the current results. The fitted value in this work for $\mathrm{Y}$ is $B_{Y}=800 \mathrm{MPa}$ (at.) ${ }^{-2 / 3}$ and together with the reported value $B_{G d}=1168 \mathrm{MPa}$ (at. $)^{-2 / 3}$ in [33], are in contrast with the fitted value of $B_{Z n}=40 \mathrm{MPa}$ $-2 / 3$ (reported as $B_{Z n}=43 \mathrm{MP}^{-2 / 3}[22]$ ) and $B_{A l}=39 \mathrm{MPa} a^{-2 / 3}$ [22], which correspond to the highest values obtained from the solid solution models.

\subsection{Grain Size}

Grain size refinement is a major handicap in $\mathrm{Mg}$ alloys. Pure as-cast $\mathrm{Mg}$ microstructures show grain sizes in the order of $1000 \mu \mathrm{m}[4,34,12]$, which display very undesirable mechanical properties. Additional thermo-mechanical treatments can lead to grain size reductions, but most Mg components are now produced by high-pressure diecasting [2]. This has encouraged deep studies on the effect of grain refinement by including additional solute elements. The effect of Zr in small quantities is remarkable, leading to grain size reductions down to 50100 $\mu \mathrm{m}[3]$. The extensive work developed by StJohn and Easton [4, 34, 3, 35] led to the well accepted Interdependence Theory [36]. Under the assumption of low thermal conductivity, this theory can also be applied to $\mathrm{Al}[37,38]$ and $\mathrm{Ti}$ alloys [38]. This theory is based on a high nucleation rate from the liquid due to the presence of solute elements controlled by constitutional undercooling. The final grain size during casting $\left(D_{g b}\right)$ is dependant on the diffusion coefficient of the respective element on $\mathrm{Mg}, D$, the growth velocity of the solid-liquid interface, $v$, the undercooling required to cause nucleation, $\Delta T$, the fraction of particles that 
successfully nucleate a grain, $f$, the total particle density, $\rho$, and a parameter called the growth restriction factor $Q=X q=X m(k-1)$, where $m$ is the slope of the liquidus in a binary phase diagram, $k$ its partition coefficient and $X$ is the solute content in the alloy, as shown in Equation (6).

$$
D_{g b}=\frac{1}{\sqrt[3]{\rho f}}+\frac{D \Delta T}{v Q}=a_{0}+\frac{a_{1}}{Q}
$$

The optimisation of $Q$ is consistent with increasing $\sigma_{Y}$, which happens for atoms with high solubility (maximising $X)$, together with $k \rightarrow 0(0<k<1)$ and $m \rightarrow \infty$. The conditions of high solubility and $k \rightarrow 0$ can be in competition, since atoms with high solubility may not have a low partition coefficient $k$. On the other hand, the condition for constitutional undercooling is that the temperature gradient is lower than $m X(1 / k-1) v / D$ [39], and higher values of $m$ and/or $1 / k$ help in the nucleation and growth of new grains.

Experimental evidence confirms this dependence of $\mathrm{Mg}$ alloys grain refinement with additions of $\mathrm{Zr}, \mathrm{Al}, \mathrm{Zn}, \mathrm{Sr}, \mathrm{Ca}$ and $\mathrm{Si}$ [3]. This approach has also been observed to apply in $\mathrm{Al}$ alloys with additions of $\mathrm{Si}, \mathrm{Sr}, \mathrm{Ti}$ and $\mathrm{Cu}$ [37]. The variation of $a_{0}$ and $a_{1}$ for the different alloying elements in $\mathrm{Mg}$ is small and the major effect lies on $Q$. Thus, its estimation is of paramount importance for describing the final grain size of $\mathrm{Mg}$ as-cast alloys.

Thermodynamics software can be used to calculate $m$ and $k$, by computing the liquidsolid transformation of each solute element on $\mathrm{Mg}$. In addition to that, CALPHAD method and updated databases of $\mathrm{Mg}$ can be applied for higher order systems to compute accurately these parameters. The results for a variety of solute elements have been compared with reported values in the literature for binary systems [4]. The values are shown in Table 1 together with the solid solubility limit on $\mathrm{Mg}$ for each element. The agreement between the computed parameters by StJohn [4] and this work is good, with the exception of Ca and $\mathrm{Si}$, which values are lower than those reported in the literature. In addition to that, 9 more elements are also included in Table 1, these have not been reported before.

Zr is the major grain refiner, with a high value of $q$. Taking into account the solubility in $\mathrm{Mg}$, the higher values of $Q$ are for $\operatorname{Zr}(79.2 \mathrm{~K}), \mathrm{Al}(50.4 \mathrm{~K}), \mathrm{Sc}(47 \mathrm{~K}), \mathrm{Ag}(40 \mathrm{~K})$ and $\mathrm{Zn}(38$ K), followed by the rare earth elements such as Gd (28 K), Ge (22 K), Y (21 K), with similar 
values to $\mathrm{Sn}(20)$. With the computed values of $m$ and $k$, the relationship between $D_{g b}$ and $1 / Q$ is depicted in Figure 4 with the fitted values of $a_{0}$ and $a_{1}$ of Equation (6), which are shown in Table 2. For Mg-Al alloys, such values correspond to data from $[4,2,19,40]$. The values of $a_{0}$ and $a_{1}$ vary from one element to another, but it is clear a distinctive behaviour of $a_{1}$ for RE elements, with much lower values than for the rest of the elements. The grain refinement of RE elements is important, even for very a low concentration of RE. From Figure 4 it is clear that the value of for the RE is much lower than others elements. The slope $a_{1}$ is dependent on $D, \Delta T$ and inverse to $v$ (Equation (6)), and it is expected that the diffusion of $\mathrm{RE}$ in $\mathrm{Mg}$ could be low due to a large atomic misfit. This parameter, $D$, frequently display large differences from one element to another, and could be the reason for the low values of $a_{1}$.

\subsection{Hall-Petch hardening parameter $k_{Y}$}

There is experimental evidence that the hardening parameter $k_{Y}$ varies with solute content in a variety of materials $[12,41,42,43]$. The dependence of this parameter on the shear modulus $\mu$ and Burgers vector $b$ is considered in different models developed in the past, such as the pile-up model by Hall [6] with $k_{Y} \propto(\mu b)^{0.5}$, the Ashby's dislocation density model [44] where $k_{Y} \propto \mu b^{0.5}$ and the flow stress model [45, 46, 47] where the dependence is $k_{Y} \propto \mu b$. Nevertheless, full understanding of the solute atoms effect on $k_{Y}$ has not been achieved. A Taylor expansion of $\mu^{m} b^{n}$ can be performed by assuming linear behaviour of both parameters in the range of the solid solubility of the alloying elements $\mu=\mu_{M g}+X \Delta \mu$ and $b=b_{M g}+X \Delta b$. By applying those models, the variation of $k_{Y}$ with solute content becomes always proportional to composition:

$$
X(\beta \Delta b+\nu \Delta \mu) \equiv X \Delta k_{Y}
$$

where $\beta$ and $\nu$ are constants dependant on the magnitude of the Burgers vector of $\mathrm{Mg}$ and shear modulus. Expressions for $\beta$ and $\nu$ are derived in the Appendix, along with its generalisation to multicomponent systems. 
From the set of experiments considered in this work, a direct study of the variation of $k_{Y}$ with composition has been performed for Mg-Zn [13, 12] and Mg-Y [11]. The results are shown in Figure 5, where the effect of $\mathrm{Zn}$ on $k_{Y}$ is noticeable. On the other hand, $k_{Y}$ has little variation with the addition of $\mathrm{Y}$. The computation of $\Delta k_{Y}$ for other elements is not direct since each solute content displays a different grain size, but it has been possible to compute by fitting the data on the systems Mg-Al [19], Sn [48] and Gd [33]. Following Equation (7) we propose the general equation:

$$
\sigma_{y}=\sigma_{f r i c}+\sigma_{p}+B_{i} X_{i}^{2 / 3}+\frac{k_{Y, M g}+X_{i} \Delta k_{Y, i}}{\sqrt{D_{g b}}}
$$

where $k_{Y, M g}=0.21\left(\mathrm{MPa} \mathrm{m}{ }^{0.5}\right)$ is the strengthening coefficient of pure $\mathrm{Mg}, X_{i}$ is the atomic solute content of element $i$ and $\Delta k_{Y, i}$ its variation on $k_{Y}$. The corresponding values of $k_{Y, i}$ are shown in Table 3 . With this complete model, both parameters $B_{i}$ and $\Delta k_{Y, i}$ are fitted simultaneously per element $i$. The computed value for $B_{G d}=900 \mathrm{MPa}$ (at.) ${ }^{-2 / 3}$ is close to $B_{G d}=1168 \mathrm{MPa}$ (at.) ${ }^{-2 / 3}$ reported at [33], although the value of $\mathrm{Al}, B_{A l}=120 \mathrm{MPa}(\text { at. })^{-2 / 3}$, varies significantly from $B_{A l}=39 \mathrm{MPa}$ at $^{-2 / 3}$ calculated at [22]. It is remarkable that the effect of $\mathrm{Zn}$ is much higher than that of any other considered element. This is not surprising if we consider the values of $\Delta b$ and $\Delta \mu$ of these elements with its effect on $k_{Y}$. The expected variation of the shear modulus $\Delta \mu$ of $\mathrm{Zn}$ is much higher than for the rest of elements, around $\Delta \mu_{Z n}=26 \mathrm{GPa} /$ at versus $\Delta \mu_{Y}=9 \mathrm{GPa} / \mathrm{at}, \Delta \mu_{A l}=9 \mathrm{GPa} / \mathrm{at}, \Delta \mu_{G d}=5 \mathrm{GPa} /$ at and $\Delta \mu_{S n}=1 \mathrm{GPa} /$ at; while the Burgers vector variation is similar in all solute elements. Thus, a higher effect on $k_{Y}$ is expected.

\section{Application of the model}

The model described in Equation (8) has been developed for binary systems. The extension to many solute elements can be performed following the approach by Gypen and Deruyttere [49, 50] for the solid solution strengthening and adding the effect of each solute in the Hall-Petch strengthening parameter $k_{Y}$, as deducted in the Appendix. The final expression becomes: 


$$
\sigma_{y}=\sigma_{f r i c}+\sigma_{p}+\sum_{i}\left(B_{i}^{3 / 2} X_{i}\right)^{2 / 3}+\frac{k_{Y, M g}+\sum_{i} X_{i} \Delta k_{Y, i}}{\sqrt{D_{g b}}}
$$

This model has been applied to pure $\mathrm{Mg}[10,11,12,14]$, the binary systems $\mathrm{Mg}-\mathrm{Al}$ [2, 19], Mg-Zn [12, 13, 29], Mg-Sn [48], Mg-Y [5, 11], Mg-Gd [33], as well as to higher order systems such as Mg-Al-Zn [51, 52], Mg-Al-Yb [53] and Mg-Y-Gd [33]. The results are shown in the experimental vs. predicted $\sigma_{Y}$ plot in Figure 6, where the legend contains each composition together with its corresponding reference. A $R^{2}=0.95$ indicates a very good agreement with the 104 data points, which covers 6 solute elements, different testing methods and thermomechanical processes.

Such model can be used to quantify the effect of each element in the solid solution on yield strenght, and to understand which contribution to the Hall-Petch relationship prevails. The relationship between $\delta_{M g}, \eta_{M g}^{\prime}$ and $\max (Q)$, i.e. the product of the maximum solubility and $q$ from Table 1, is shown in Figure 7 for various solute elements. By taking into account the elements with enough solubility to display a certain effect on the grain size, there are clear trends relating $\max (Q)$ with $\delta_{M g}$ and $\eta_{M g}^{\prime}$. This is depicted in the projections onto the planes $\max (Q)$ vs. $\eta_{M g}^{\prime}$ (in red) and $\max (Q)$ vs. $\delta_{M g}$ (in green). The more the elastic misfit, the more is the expected growth restriction factor, which increases also with low values of atomic misfit. Thus, small atoms with high shear modulus seem to help in controlling grain size. That means that, during the liquid-solid transformation, the solute concentration in the liquid is close to the solute content of the material $X$, which is more affected by the atomic misfit, since the shear modulus does not exist in the liquid, but also the solute concentration in the solid is much lower than $X$, where $\mu$ could also play a role. On the other hand, large atoms with low shear modulus have little effect on grain size.

A flow chart illustrating the design process of $\mathrm{Mg}$ alloys is depicted in Figure 8, where the physical parameters and models are related to the final value of $\sigma_{Y}$. The main physical parameters are the Burgers vector, $b$, the shear modulus, $\mu$, and the thermodynamic magnitudes $m$ and $q$. The parameters used to compute $a_{0}$ and $a_{1}$ (i.e. $D, v, \Delta T, \rho$ and $f$ ), are of lower importance, since the dependence of $a_{0}$ and $a_{1}$ depicted in Figure 4 show little variation 
from one element to another, apart for the RE elements, which form a separate group. There is a common dependence of $B_{i}$ and $k_{Y}$ on the parameters $b$ and $\mu$. This dependence is better understood for $B_{i}$, with Fleisher's and/or Labusch's approaches, than for $k_{Y}$. Even so, from the results shown in this work, $B_{i}$ is more related to the atomic misfit, while $k_{Y}$ seems to be more dependant on the elastic misfit. As elastic and atomic size misfits show certain inverse relationship (see Figure 3), it is not surprising that $B_{i}$ and $k_{Y}$ are inversely related, as suggested by the data shown in this work. This is highlighted in the fitted data shown Table 3 for $B_{i}$ and $k_{Y}$. The large value of $\mathrm{Zn}$ on $\Delta k_{Y}$ is in contrast with its low solid solution hardening effect, while the opposite is true, especially for RE elements, with large values of $B_{i}$ and negligible variation on $k_{Y}$. This demands a compromise between $B_{i}$ and $k_{Y}$, since both cannot be simultaneously optimised. On the other hand, from the results displayed in Figure 7, the growth restriction factor parameter, $Q$, is also related with atomic and elastic misfit. Thus, fine grains are expected for solute atoms with with higher elastic misfit and low atomic size misfit. It is clear that, $b$ and $\mu$ are key parameters in the design of new alloys.

\section{Conclusions}

The combination of several experimental works and theoretical models found in the literature lead to a general approach for the prediction of yield strength $\sigma_{Y}$ on $\mathrm{Mg}$ alloys under the influence of solute elements. The Hall-Petch relationship has been analysed and divided in different contributions which dependence on concentration has been examined.

It has been shown how both the atomic size and elastic misfit control the solid solution hardening and the strengthening coefficient on Hall-Petch relationship. The calculation results suggest that the atomic size misfit has a larger effect on the solid solution hardening, and that positive values of $\delta$ around 1-1.5 could have a remarkable effect on this factor. This has seen to be true in Mg-alloys, where this region of $\delta$ is covered by RE elements, and also supported in Cu-alloys. Many attempts to explain the effect of RE element on Mg-alloys have been made, but complete understanding is not achieved and further investigations are required. 
The variation of $k_{Y}$ is also dependent on the atomic size and elastic misfits, although the effect of the difference on shear modulus seems to have a larger effect than the atomic size misfit. As a guideline for alloy design, large variations of $\delta$ due to different atomic sizes will affect strongly the solid solution hardening, while a variation on the shear modulus is more related to the strengthening parameter $k_{Y}$.

Grain size displays very different dependence on the solute content, where thermodynamics plays now an important role. Elements with deep eutectics and high solubility will lead to finer grains. However, some of the parameters controlling this process are difficult to estimate, such as $D$ and $v$ on Equation (6), and some extent of experimental fitting is required. Nevertheless, a functionality with atomic size and/or shear modulus of the solute has been seen, where the maximum grain refinement seems to be correlated with small atoms and large shear modulus.

There has traditionally been a large effort on optimizing $D_{g b}$ above the rest of factors on the Hall-Petch relationship. Its effect is larger than the rest of factors and once a chemical composition is defined, no further influence can be induced on $\Delta \sigma_{Y}$ or $k_{Y}$. In the case of as cast materials, the parameter to optimize is $\Delta \sigma_{Y} / \Delta X$ which comprises $D_{g b}$. The results obtained in this work support, the relevace of $D_{g b}$ refinement, because it has a larger effect on $\sigma_{Y}$, but it is clear that some elements such us Zn, improve $\sigma_{Y}$ much more efficiently than others due to a large effect on $k_{Y}$. To illustrate this effect, same grain sizes in $\mathrm{Mg}$ and in Mg-2.3Zn display a factor of 2 in $\sigma_{Y}$ (see Figure 5) where the same effect for grain size straightening is only possible for a reduction of a factor of 4 in mean grain size. The total optimization of mechanical properties goes through the total understanding of the effect of these elements in $\mathrm{Mg}$ alloys.

\section{Acknowledgements}

The authors wish to acknowledge financial support from the Accelerated Metallurgy Project, which is co-funded by the European Commission in the 7th Framework Programme (Contract NMP4-LA-2011-263206), by the European Space Agency and by the individual partner 
organisations.

\section{Apendix}

The Taylor expansion of $(\mu b)^{1 / 2}$ from the pile-up model by Hall [6] takes the form:

$\sqrt{\mu b}=\sqrt{\mu_{M g}+X \Delta \mu} \sqrt{b_{M g}+X \Delta b}=\sqrt{\mu_{M g} b_{M g}}+\left(\Delta \mu \frac{1}{2} \sqrt{\frac{b_{M g}}{\mu_{M g}}}+\Delta b \frac{1}{2} \sqrt{\frac{\mu_{M g}}{b_{M g}}}\right) X+O\left[X^{2}\right]$

Thus, the variation of the strengthening parameter $k_{Y}$ is $\Delta k_{Y}=\nu \Delta \mu+\beta \Delta b$ where

$$
\nu=\frac{1}{2} \sqrt{\frac{b_{M g}}{\mu_{M g}}} \quad ; \quad \beta=\frac{1}{2} \sqrt{\frac{\mu_{M g}}{b_{M g}}}
$$

By using the dislocation density model proposed by Ashby:

$\mu \sqrt{b}=\left(\mu_{M g}+X \Delta \mu\right) \sqrt{\left(b_{M g}+X \Delta b\right)}=\mu_{M g} \sqrt{b_{M g}}+\left(\Delta \mu \sqrt{b_{M g}}+\Delta b \frac{\mu_{M g}}{2} \sqrt{\frac{1}{b_{M g}}}\right) X+O\left[X^{2}\right]$

It follows that the parameters $\nu$ and $\beta$ are:

$$
\nu=\sqrt{b_{M g}} \quad ; \quad \beta=\frac{\mu_{M g}}{2} \sqrt{\frac{1}{b_{M g}}}
$$

In the case of Flow stress model, the Taylor expansion becomes simple:

$$
\mu b=\left(\mu_{M g}+X \Delta \mu\right)\left(b_{M g}+X \Delta b\right)=\mu_{M g} b_{M g}+\left(\Delta \mu b_{M g}+\Delta b \mu_{M g}\right) X+O\left[X^{2}\right]
$$

and then the parameters $\nu$ and $\beta$ are:

$$
\nu=b_{M g} \quad ; \quad \beta=\mu_{M g}
$$

By doing simple mathematical calculations, the generalisation to many solute elements 
takes the form of any of the three possibilities is:

$$
\mu^{p} b^{q}=\left(\mu_{M g}+\sum_{i} X_{i} \Delta \mu_{i}\right)^{p}\left(b_{M g}+\sum_{i} X \Delta b_{i}\right)^{q}=\mu_{M g}^{p} b_{M g}^{q}+\sum_{i}\left(\Delta \mu_{i} \nu+\Delta b_{i} \beta\right) X_{i}+O\left[X^{2}\right]
$$

where $p=q=1 / 2$ for Hall's model, $p=1$ and $q=1 / 2$ for Ashby model and $p=1$ and $q=1$ for Flow stress model, and $\nu$ and $\beta$ corresponds to the expressions computed in each case. Thus, the dependence of $k_{Y}$ with respect to more than one solute element will always be a linear combination of each individual element:

$$
k_{Y}=k_{Y, M g}+\sum_{i} X_{i} \Delta k_{Y, i}
$$




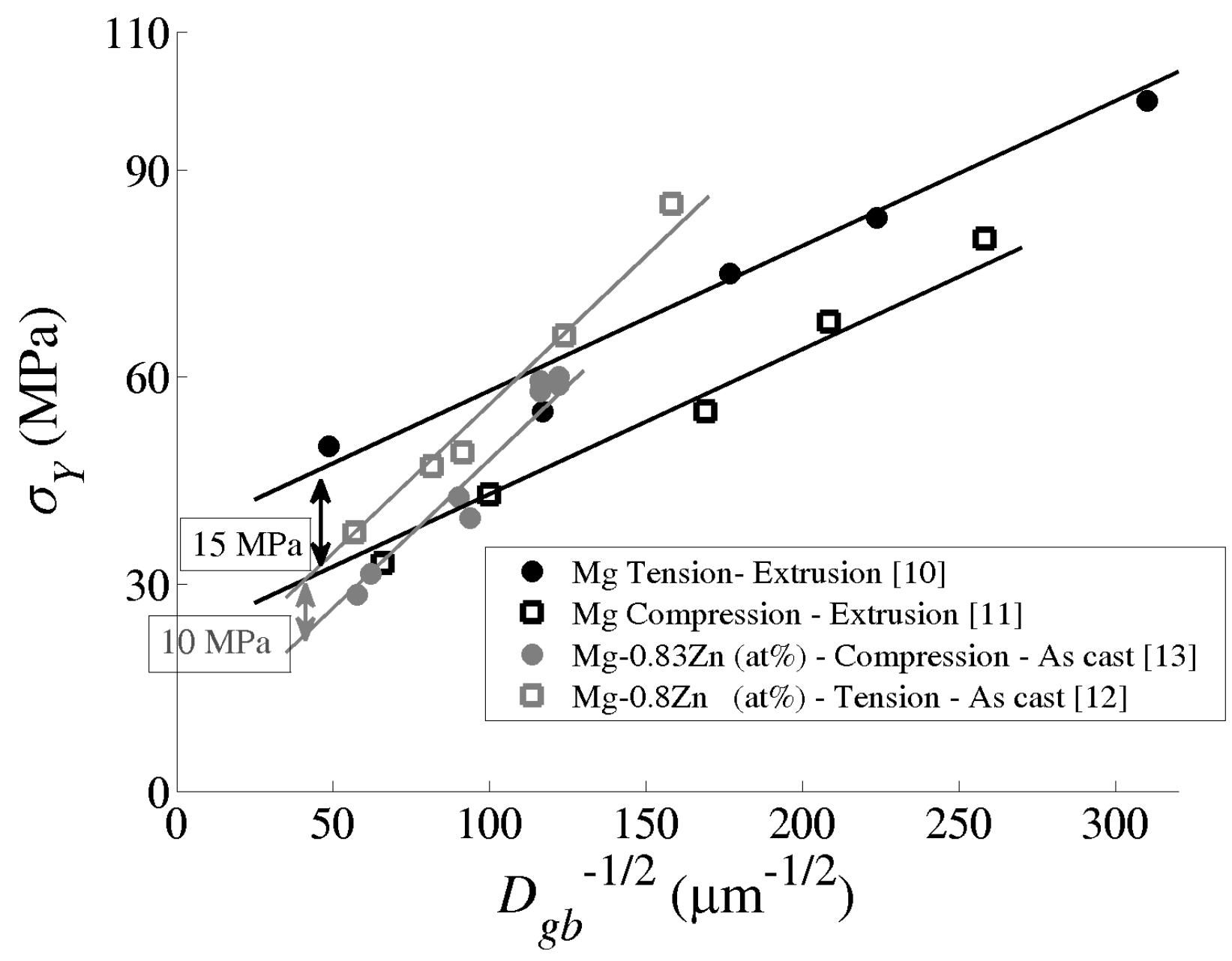

Figure 1: Comparison of Hall-Petch plots of different testing methodology for similar composition and thermo-mechanical treatment. 


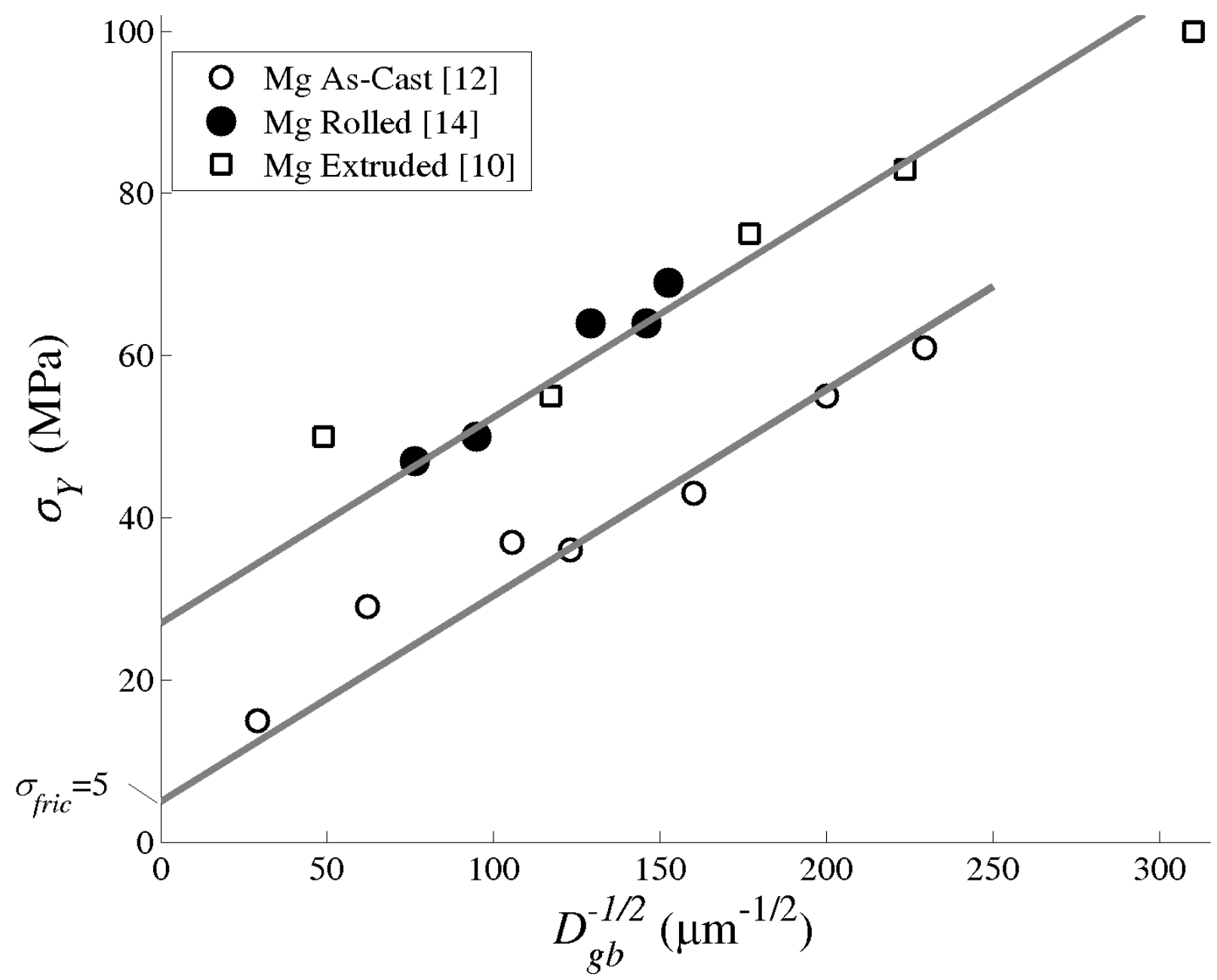

Figure 2: Mechanical processing and effect of $\sigma_{Y}$ on as-cast, rolled and extruded Mg 


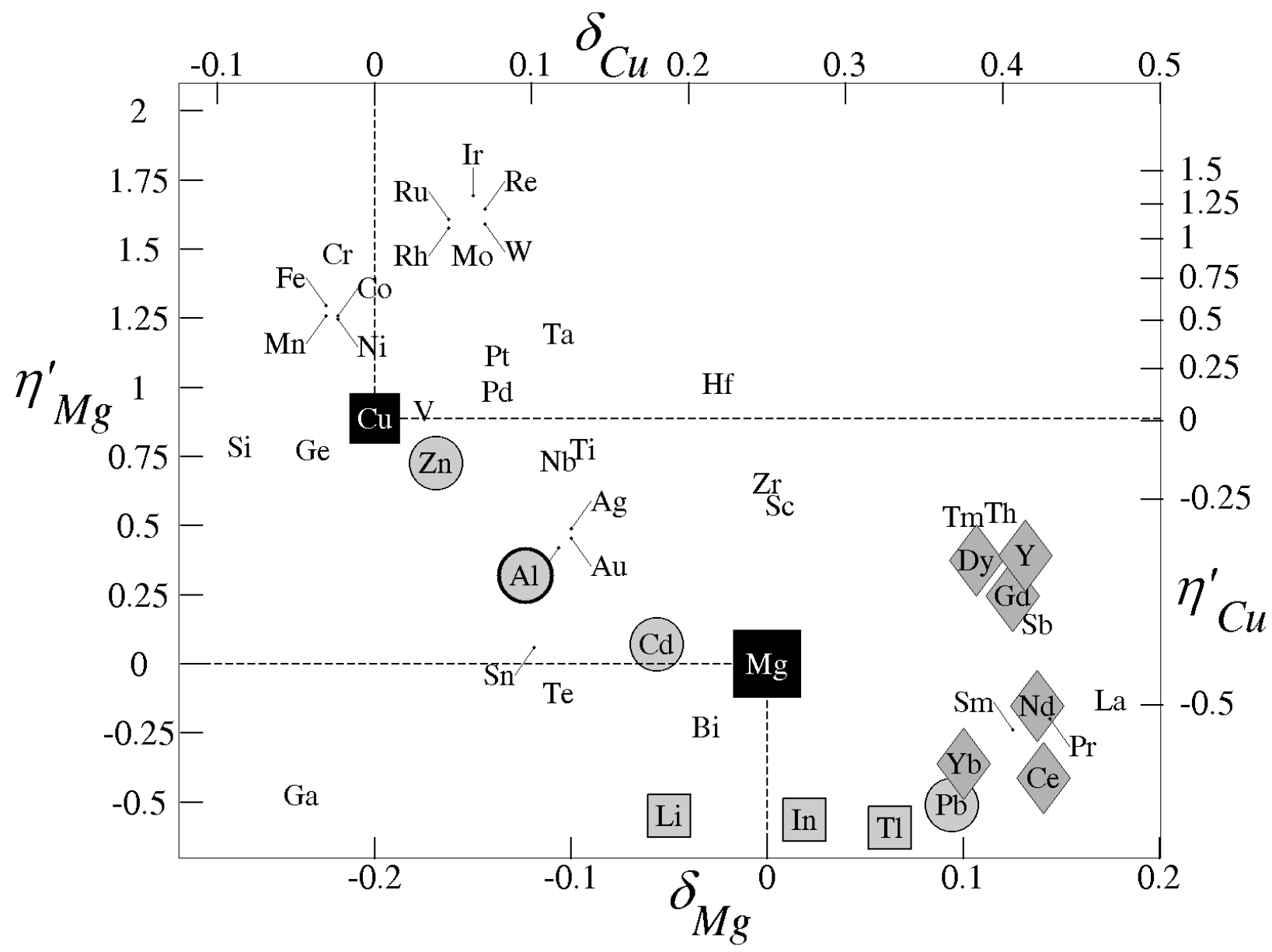

Figure 3: Elastic $\left(\eta_{M g}^{\prime}, \eta_{C u}^{\prime}\right)$ and atomic size $\left(\delta_{M g}, \delta_{C u}\right)$ misfits for Mg-solute and Cu-solute interactions. Elements highlighted in grey squares corresponds to negligible solid solution hardening effect in Mg alloys, while elements highlighted in grey circles correspond to larger effects and grey rhombi corresponds to elements with remarkable hardening, which can not be explained with the proposed models. 


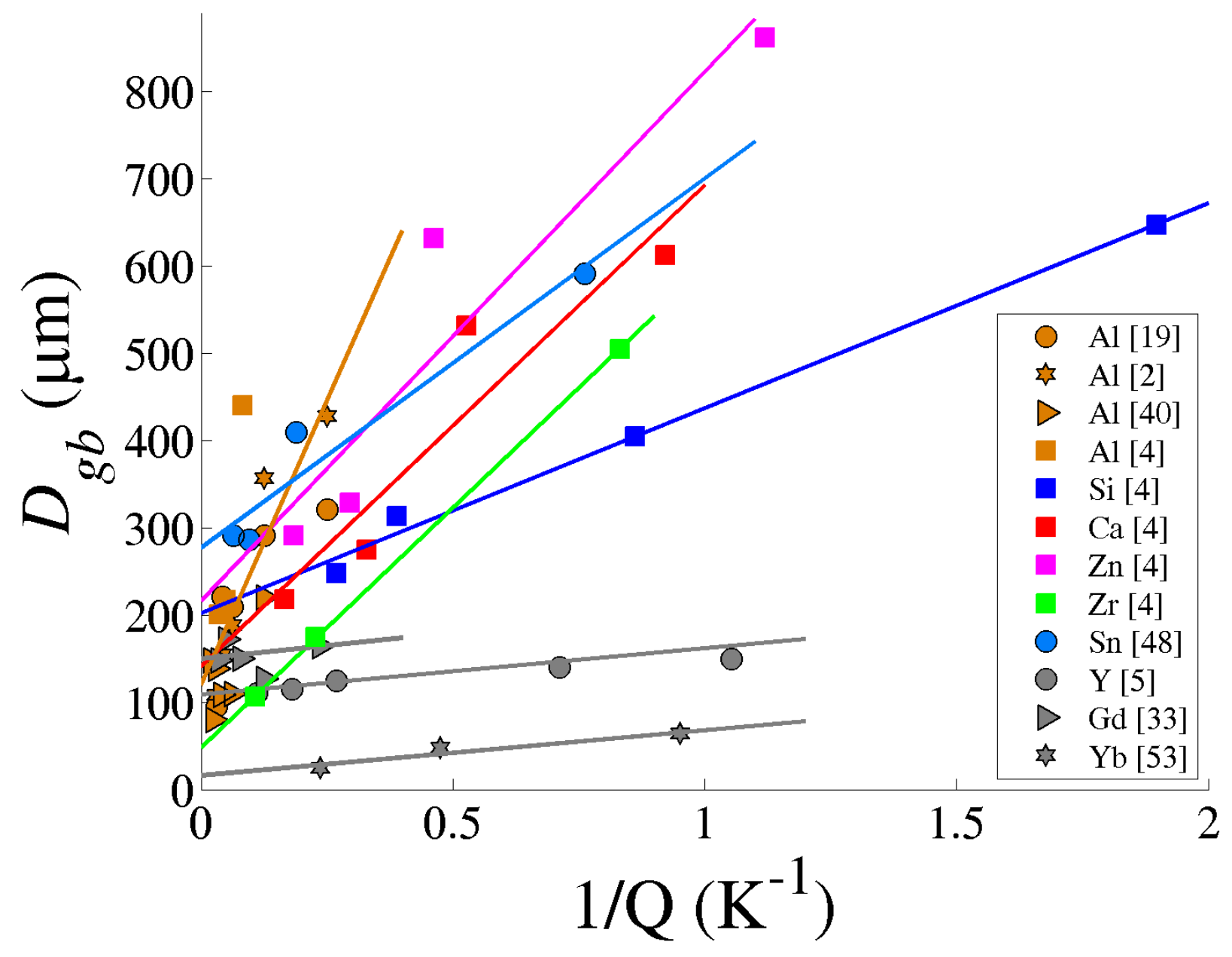

Figure 4: Relation of $D_{g b}$ grain size with the inverse of the Growth Restriction Factor, for the elements Al, Si, Ca, Zn, Zr, Sn, Y, Gd and Yb. Lines are fitted by the least square method. Data points for $\mathrm{Yb}$ corresponds to $\mathrm{Mg}-\mathrm{Al}-\mathrm{Yb}$ alloys with $\mathrm{Al}$ constant, then $Q$ is computed with respect to $\mathrm{Yb}$. 


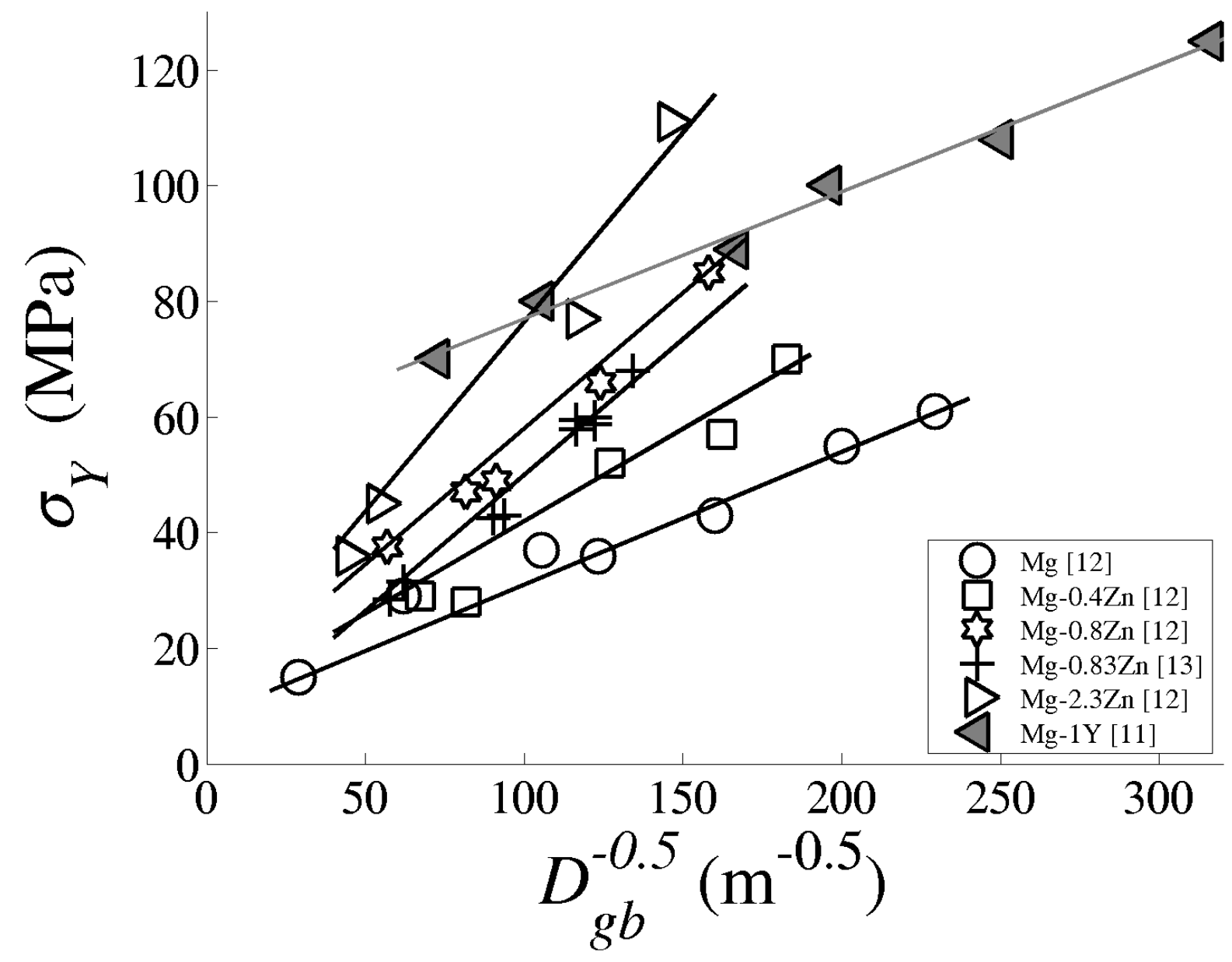

Figure 5: Hall-Petch plots for the effect of the solute elements $\mathrm{Zn}$ and $\mathrm{Y}$ on $k_{Y}$. Mg and $\mathrm{Mg}-\mathrm{Zn}$ data points correspond to as cast materials $\mathrm{Mg}, \mathrm{Mg}-0.4, \mathrm{Mg}-0.8 \mathrm{Zn}$ and $\mathrm{Mg}-2.3 \mathrm{Zn}$ in tensile testing from [12] and in compressive testing Mg-0.83Zn from [13], while $\mathrm{Mg}-\mathrm{Y}$ data points were extruded measured in compressive tests [11]. 


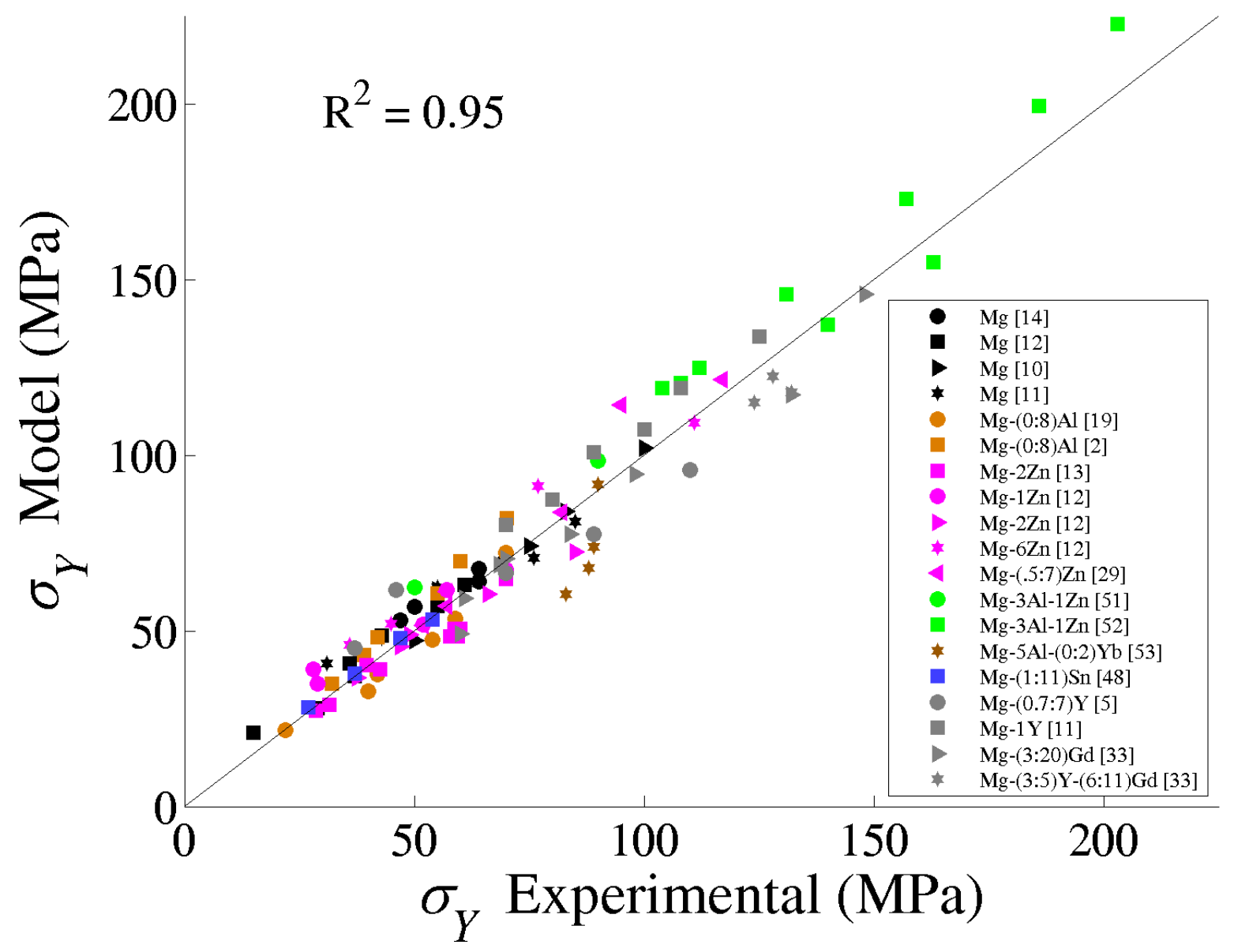

Figure 6: Experimental vs. prediction model of Equation (9). The composition and its corresponding reference are indicated in the legend. For set of experiments with a variation on the solute content, the notation $\left(X_{1}: X_{2}\right)$ shows its minimum $X_{1}$ and maximum $X_{2}$ values. Alloy contents are reported in wt\%. Datasets corresponding to Mg-Zn, from references $[13,12]$ contains small additions of $\mathrm{Zr}$ inducing grain size variation. Its effect as solute on $\Delta \sigma_{Y}$ and $k_{Y}$ is considered negligible. 


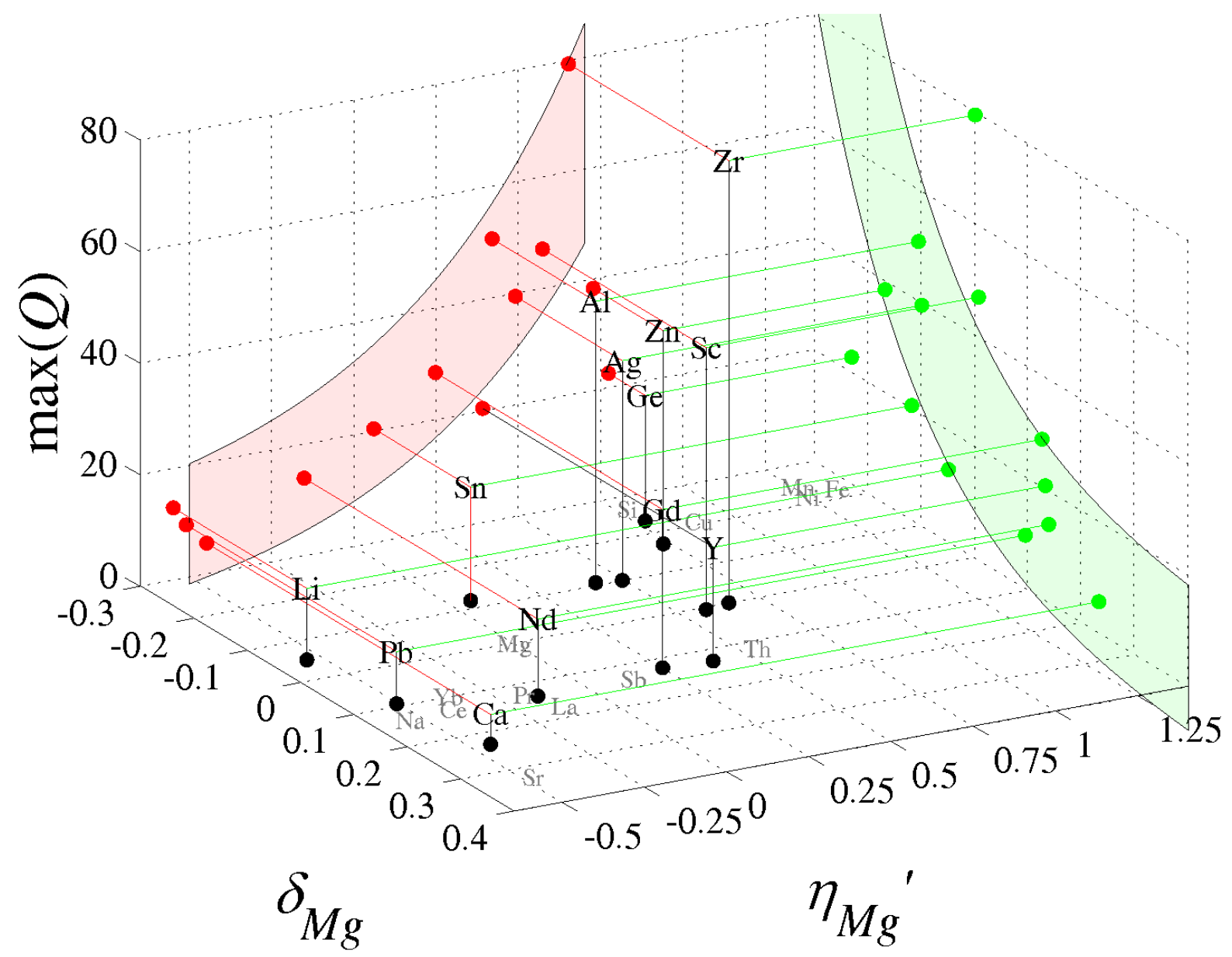

Figure 7: Relationship between elastic misfits $\eta_{M g}^{\prime}$, atomic size misfits $\delta_{M g}$ and maximum $Q$ for each element. Elements with refining effect are shown with projections onto $\left(\delta_{M g}, \max (Q)\right)$ and $\left(\eta_{M g}^{\prime}, \max (Q)\right)$ planes. Elements with low influence on grain refinement are depicted in grey and have $\max (Q) \approx 0$ 


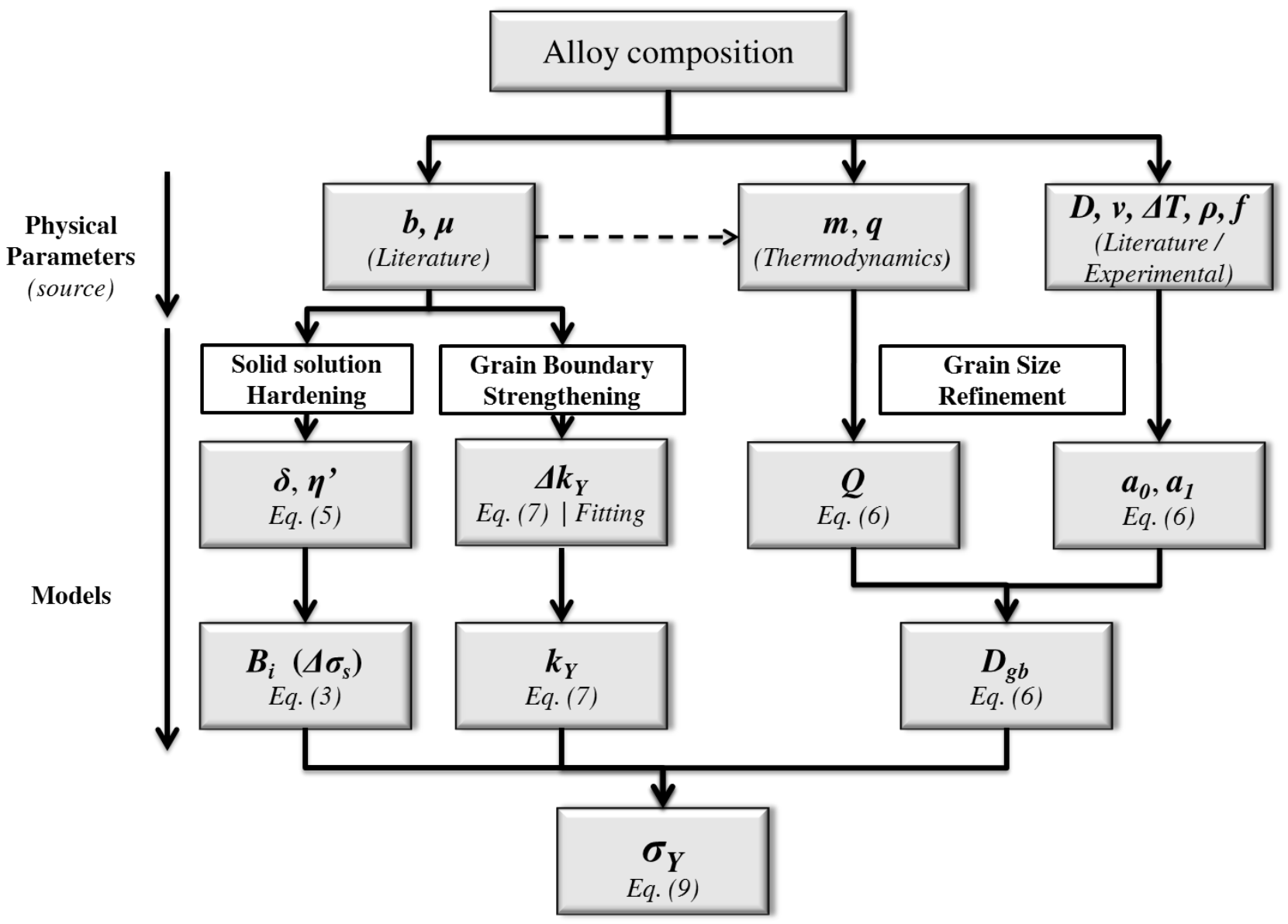

Figure 8: Design flow chart for new Mg alloys 
Table 1: Solubility and growth restriction factor parameters $m, k$ and $m(k-1)$, of various alloying elements in $\mathrm{Mg}$.

\begin{tabular}{|c|c|c|c|c|c|c|c|c|}
\hline \multirow[b]{2}{*}{ Element } & \multirow{2}{*}{$\begin{array}{c}\text { Solubility } \\
\text { (wt\%) }\end{array}$} & \multirow{2}{*}{\multicolumn{2}{|c|}{$\begin{array}{l}m \\
\text { This Work }\end{array}$}} & \multicolumn{2}{|r|}{$k$} & \multicolumn{2}{|c|}{$q=m(k-1)$} & \multirow[b]{2}{*}{ System } \\
\hline & & & & {$[4]$} & This Work & {$[4]$} & This Work & \\
\hline $\mathrm{Ag}$ & 13.8 & & -4.3 & & 0.34 & & 2.8 & eutectic \\
\hline $\mathrm{Al}$ & 12.7 & -6.9 & -6.4 & 0.37 & 0.38 & 4.3 & 3.9 & eutectic \\
\hline $\mathrm{Ca}$ & 0.7 & -12.7 & -8.2 & 0.06 & 0.04 & 12 & 7.8 & eutectic \\
\hline $\mathrm{Ce}$ & 0.3 & -2.9 & -2.9 & 0.04 & 0.01 & 2.7 & 2.8 & eutectic \\
\hline $\mathrm{Cu}$ & 0.13 & -5.4 & -4.9 & 0.02 & $\approx 0$ & 5.3 & 4.8 & eutectic \\
\hline $\mathrm{Fe}$ & $\approx 0$ & -5.5 & -5.6 & 0.054 & 0.06 & 5.2 & 5.3 & eutectic \\
\hline Gd & 20.2 & & -3.4 & & 0.59 & & 1.4 & eutectic \\
\hline Ge & $21.6^{*}$ & -4.4 & $-2.2^{*}$ & 0 & $0.53^{*}$ & 4.4 & $1.04^{*}$ & eutectic \\
\hline $\mathrm{K}$ & $58.2^{*}$ & & $-5.0^{*}$ & & $0.68^{*}$ & & $1.5^{*}$ & eutectic \\
\hline $\mathrm{La}$ & 0.06 & & -2.6 & & $\approx 0$ & & 2.5 & eutectic \\
\hline $\mathrm{Li}$ & 5.6 & & -8.1 & & 0.72 & & 2.2 & eutectic \\
\hline $\mathrm{Mn}$ & 1.9 & 1.5 & 0.3 & 1.1 & 1.13 & 0.15 & 0.04 & peritectic \\
\hline $\mathrm{Na}$ & 0.3 & & -6.6 & & 0.15 & & 5.5 & eutectic \\
\hline $\mathrm{Nd}$ & 4.8 & & -3.5 & & 0.17 & & 2.8 & eutectic \\
\hline $\mathrm{Ni}$ & 0 & -6.1 & -6.1 & 0 & $\approx 0$ & 6.1 & 6.1 & eutectic \\
\hline $\mathrm{Pb}$ & $41^{*}$ & -2.8 & $-3.1^{*}$ & 0.62 & $0.97^{*}$ & 1 & $0.1^{*}$ & eutectic \\
\hline $\operatorname{Pr}$ & 0.4 & & -3.0 & & 0.01 & & 2.9 & eutectic \\
\hline $\mathrm{Sb}$ & $0^{*}$ & -0.5 & $-1.0^{*}$ & 0 & $0^{*}$ & 0.53 & $0.9^{*}$ & eutectic \\
\hline $\mathrm{Sc}$ & $12.1^{*}$ & 4.0 & $5.0^{*}$ & 1.65 & $1.78^{*}$ & 2.6 & $3.8^{*}$ & peritectic \\
\hline $\mathrm{Si}$ & $\approx 0$ & -9.3 & -7.6 & 0 & 0.00 & 9.2 & 7.5 & eutectic \\
\hline Sn & 13.9 & -2.4 & -2.3 & 0.39 & 0.37 & 1.5 & 1.4 & eutectic \\
\hline $\mathrm{Sr}$ & 0.3 & -3.5 & -.4 & 0.006 & 0.02 & 3.5 & 3.3 & eutectic \\
\hline Th & 0 & & -1.4 & & $\approx 0$ & & 1.4 & eutectic \\
\hline $\mathrm{Y}$ & 12.1 & -3.4 & -3.2 & 0.5 & 0.48 & 1.7 & 1.6 & eutectic \\
\hline $\mathrm{Yb}$ & $0^{*}$ & -3.1 & $-2.1^{*}$ & 0.17 & $0^{*}$ & 2.5 & $2.1^{*}$ & eutectic \\
\hline $\mathrm{Zn}$ & 7.5 & -6.0 & -5.9 & 0.12 & 0.14 & 5.3 & 5 & eutectic \\
\hline $\mathrm{Zr}$ & 2.7 & 6.9 & 8.0 & 6.55 & 4.70 & 38.3 & 29.5 & peritectic \\
\hline
\end{tabular}

*Binary systems not critically assessed 
Table 2: Fitted values of $a_{0}$ and $a_{1}$ for different elements

\begin{tabular}{l|cc} 
Element & $a_{0}(\mu \mathrm{m})$ & $a_{1}(\mu \mathrm{m} \cdot \mathrm{K})$ \\
\hline $\mathrm{Al}$ & 120 & 1300 \\
$\mathrm{Al}[4]$ & 95.9 & 3534 \\
$\mathrm{Si}[4]$ & 202 & 290 \\
$\mathrm{Ca}[4]$ & 135 & 864 \\
$\mathrm{Zn}[4]$ & 201 & 668 \\
$\mathrm{Zr}[4]$ & 52.5 & 695 \\
$\mathrm{Sn}$ & 276 & 423 \\
$\mathrm{Y}$ & 110 & 53 \\
$\mathrm{Gd}$ & 150 & 60 \\
Yb* & 16 & 52 \\
\hline
\end{tabular}

${ }^{*}$ Fitting performed with $\mathrm{Mg}-\mathrm{Al}-\mathrm{Yb}$ alloys with $\mathrm{Al}$ constant. 
Table 3: Variation of the strengthening parameter $k_{Y}$ in $\mathrm{Mg}$ alloys in presence of various solute elements

\begin{tabular}{c|cc} 
& $B$ & $\Delta K_{Y}$ \\
Element & $\left(\mathrm{MPa} \mathrm{at}^{-2 / 3}\right)$ & $\left(\mathrm{MPa} \mathrm{m}^{0.5} \mathrm{at}^{-1}\right)$ \\
\hline $\mathrm{Zn}$ & 40 & 18 \\
$\mathrm{Y}$ & 800 & 1 \\
$\mathrm{Al}$ & 120 & 2 \\
$\mathrm{Sn}$ & 280 & 3 \\
$\mathrm{Gd}$ & 900 & 5 \\
\hline
\end{tabular}




\section{References}

[1] Toda-Caraballo I, Galindo-Nava EI, Rivera-Díaz-del-Castillo PEJ. J Alloys Compd 2013;266:217.

[2] Polmear I. Light Alloys, From Traditional Alloys to Nanocrystals. Elsevier Ltd., Oxford 2006.

[3] Lee YC, Dahle AK, StJohn DH. Metall Mater Trans A 2000;31A:2895.

[4] StJohn DH, Qian M, Easton AM, Cao P, Hildebrand Z. Metall Mater Trans A 2005;36A:1669.

[5] Gao L, Chen RS, Han EH. J Alloys Compd 2009;472:234.

[6] Hall EO. P Phys Soc B 1951;64:747-.

[7] Petch NJ. J Iron Steel I 1953;174:25.

[8] Galindo-Nava EI, Rivera-Díaz-del-Castillo PEJ. Int J Plasticity 2013;In press.

[9] Bhattacharya B. Plastic Deformation Behavior of pure Pure Magnesium in the Temperature Range 4.2K-300K. PhD thesisMcMaster University, Hamilton, Ontario 2006.

[10] Wilson DV, Chapman JA. Philos Mag 1963;8:1543.

[11] Somekawa H, Mukai T. Mater Sci Eng A 2013;561:378.

[12] Caceres CH, Mann GE, Griffiths JR. Metall Mater Trans A 2011;42A:1950.

[13] Mann GE, Griffiths JR, Caceres CH. J Alloys Compd 2004;378A:188.

[14] Ono N, Nowak R, Miura S. Mater Lett 2004;58:39.

[15] Mott NF, Nabarro FRN. Phys Soc Bristol Conference Report 1948:1.

[16] Fleischer RL. Acta Metall Mater 1963;11:203. 
[17] Kocks UF, al AS Argon. Thermodynamics and Kinetics of Slip. Progress in Materials Science. Pergamon press, Oxford 1975.

[18] Labusch R. Phys status solidi 1970;41:659.

[19] Caceres CH, Rovera DM. J Light Met 2001;1:151.

[20] Lukáč P. Phys status solidi (A) 1992;131:337.

[21] Čižek L. J Mater Sci 1974;9:1517.

[22] Akhtar A, Teghtsoonian E. Philos Mag 1972;25A:897.

[23] Caillard D, Martin JL. Thermally activated mechanisms in crystal plasticity. Pergamon, Oxford 2003.

[24] Mordike BL. Mater Sci Eng A 2002;324:103.

[25] Chino Y, Kado M, Mabuchi M. Acta Mater 2008;56:387.

[26] Miura S, Imagawa S, Toyoda T, Ohkubo K, Mohri T. Mater Trans 2008;49(5):952.

[27] Ninomiya R, Yukawa H, Morinaga M, Kubota K. J Alloys Compd 1994;215:315.

[28] Le QC, Zhang ZQ, Cui ZW Shao JZ, Xie Y. T Nonferr Metal Soc 2010;20:352.

[29] Caceres CH, Blake A. Phys status solidi 2002;194(1):147.

[30] Stanford N, Barnett MR. Int J Plasticity 2013;47:165-181.

[31] Liu H, Chen Y, Zhao H, Wei S, Gao W. J Alloys Compd 2010;504:345.

[32] Gao L, Zhou J, Sun ZM, Chen RS, Han EH. Chinese Sci Bull 2011;56:1038.

[33] Gao L, Chen RS, Han EH. J Alloys Compd 2009;481:379-384.

[34] StJohn DH, Easton MA, Qian M, Taylor JA. Metall Mater Trans A 2013;44A:2935.

[35] Easton MA, StJohn DH. Acta Mater 2001;49:1867. 
[36] StJohn DH, Qian M, Easton MA, Cao P. Acta Mater 2011;59:4907.

[37] Mitrăsinović AM, Hernández FC Robles. Mater Sci Eng 2012;540:63.

[38] StJohn DH, Easton MA, Qian M, Cao P, Birmingham MJ. Mater Sci Forum 2011;690:206.

[39] RS Feigelson ed. 50 years of Progress in Crystal Growth. Elsevier BV, Amsterdam 2004.

[40] Fox FA. J I Met 1945;71:415.

[41] Nakada AY, Keh AS. Metall Trans 1971;2:441.

[42] Nagarjuna S, Srinivas M, Balasubramanian K, Sarma DS. Acta Mater 1996;44(6):2285.

[43] Mintz B, Gunawardana D, Su H. Mater Sci Tech 2008;24:596.

[44] Ashby MF. Philos Mag 1970;21:399.

[45] Hirth JP. Metall Trans 1972;3:3047.

[46] Thompson AW, Baskes MI. Philos Mag 1973;28:301.

[47] Meyers MA, Ashworth E. Philos Mag 1982;46:737.

[48] Shi BQ, Chena RS, Kea W. J Alloys Compd 2011;509:3357.

[49] Gypen LA, Deruyttere A. J Mater Sci 1977;12:1028.

[50] Gypen LA, Deruyttere A. J Mater Sci 1977;12:1034.

[51] Li Y, Enoki M. Mater Trans 2007;48(9):2343.

[52] Barnett MR, Keshavarz Z, Beer AG, Atwell D. Acta Mater 2004;52(17):5093.

[53] Jo SM, Park KC, Kim BH, Kimura H, Park K, Park YH. Mater Trans 2011;52:6. 